\title{
elsevier_PHYTO_10940
}

\section{Characterisation of triterpenes and new phenolic lipids in Cameroonian propolis}

M.N. Kardar

T. Zhanga

G.D. Coxon ${ }^{\mathrm{a}}$

D.G. Watson ${ }^{\mathrm{a}}$

J. Fearnley ${ }^{\mathrm{b}}$

V. Seidela,*

veronique.seidel@strath.ac.uk

aStrathclyde Institute of Pharmacy and Biomedical Sciences, University of Strathclyde, Glasgow G4 ORE, Scotland, UK

${ }^{b}$ Apiceutical Research Centre, Whitby, North Yorkshire, UK

${ }^{*}$ Corresponding author. Address: Pharmacognosy/Natural Products Drug Discovery Research Group, Strathclyde Institute of Pharmacy and Biomedical Sciences, University of Strathclyde, 161 Cathedral street, Glasgow G4 ORE, UK. Tel.: +44(0)141548 2751; fax: +44(0)141 5522562 .

\section{Abstract}

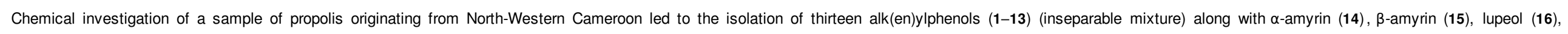

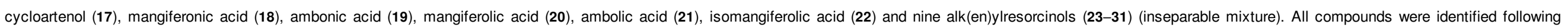

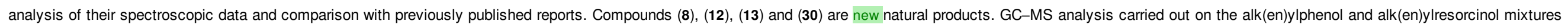

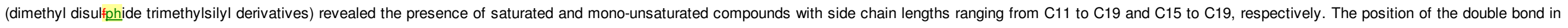

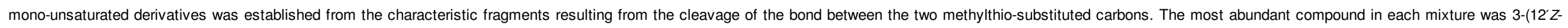

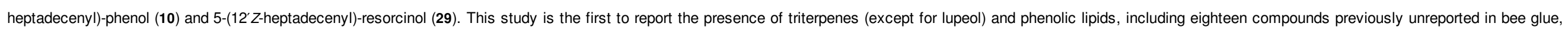
in an African sample.

Keywords: Cameroonian propolis; Triterpenes; Alk(en)ylphenols; Alk(en)ylresorcinols; Phenolic lipids

\section{Introduction}

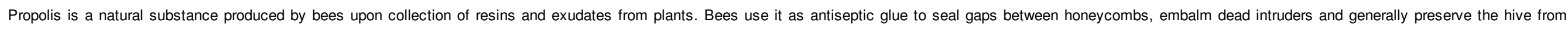

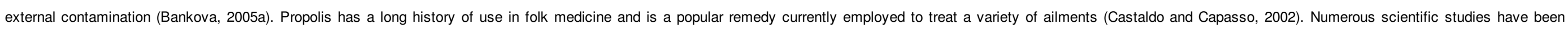

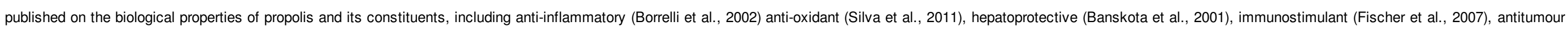
(Sawicka et al., 2012), neuroprotective (Nakajima et al., 2009) and antimicrobial activity (Seidel et al., 2008).

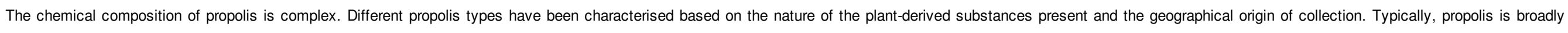

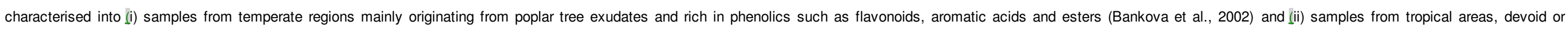

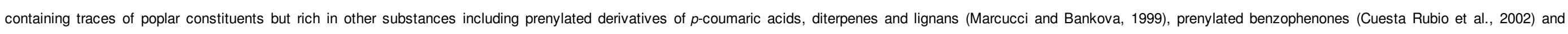
prenylated flavonoids (Raghukumar et al., 2010). 


\section{elsevier_PHYTO_10940}

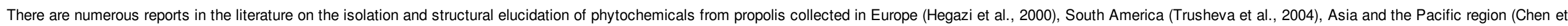

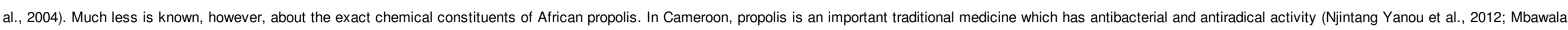

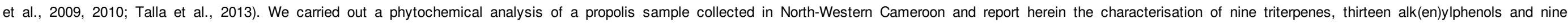
alk(en)ylresorcinols.

\section{Results and discussion}

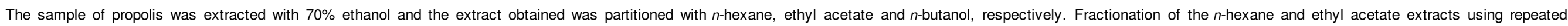

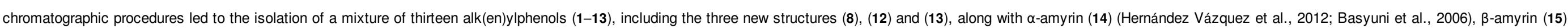

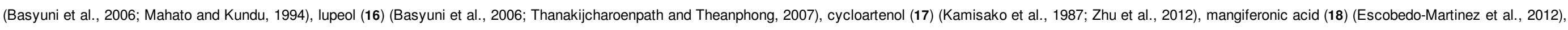

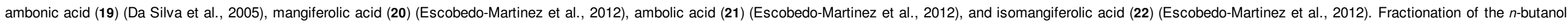

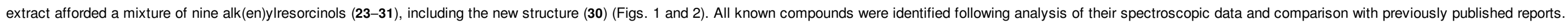

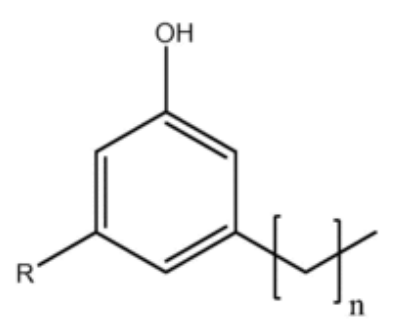

$$
\begin{aligned}
& \mathrm{n}=10, \mathrm{R}=\mathrm{H}(\mathbf{1}) \\
& \mathrm{n}=13, \mathrm{R}=\mathrm{H}(\mathbf{2}) \\
& \mathrm{n}=14, \mathrm{R}=\mathrm{H}(\mathbf{3}) ; \mathrm{R}=\mathrm{OH}(\mathbf{2 3}) \\
& \mathrm{n}=15, \mathrm{R}=\mathrm{H}(\mathbf{4}) ; \mathrm{R}=\mathrm{OH}(\mathbf{2 4}) \\
& \mathrm{n}=16, \mathrm{R}=\mathrm{H}(\mathbf{5}) ; \mathrm{R}=\mathrm{OH}(\mathbf{2 5}) \\
& \mathrm{n}=18, \mathrm{R}=\mathrm{H}(\mathbf{6})
\end{aligned}
$$

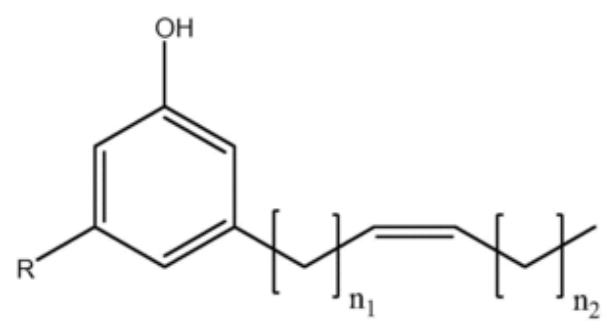

$\mathrm{n}_{1}=9, \mathrm{n}_{2}=3, \mathrm{R}=\mathrm{H}(\mathbf{7}) ; \mathrm{R}=\mathrm{OH}(\mathbf{2 6}$ $\mathrm{n}_{1}=11, \mathrm{n}_{2}=1, \mathrm{R}=\mathrm{H}(\mathbf{8})$

$\mathrm{n}_{1}=7, \mathrm{n}_{2}=7, \mathrm{R}=\mathrm{H}(\mathbf{9}) ; \mathrm{R}=\mathrm{OH}$ (27)

$\mathrm{n}_{1}=11, \mathrm{n}_{2}=3, \mathrm{R}=\mathrm{H}(\mathbf{1 0}) ; \mathrm{R}=\mathrm{OH}(\mathbf{2 9})$

$\mathrm{n}_{1}=13, \mathrm{n}_{2}=1, \mathrm{R}=\mathrm{H}(\mathbf{1 1}) ; \mathrm{R}=\mathrm{OH}(\mathbf{3 0})$

$\mathrm{n}_{1}=12, \mathrm{n}_{2}=4, \mathrm{R}=\mathrm{H}(\mathbf{1 2})$

$\mathrm{n}_{1}=13, \mathrm{n}_{2}=3, \mathrm{R}=\mathrm{H}(\mathbf{1 3}) ; \mathrm{R}=\mathrm{OH}(\mathbf{3 1})$

$\mathrm{n}_{1}=10, \mathrm{n}_{2}=4, \mathrm{R}=\mathrm{OH}(\mathbf{2 8})$

Fig. 1 Structures of alk(en)ylphenols and alk(en)ylresorcinols in Cameroonian propolis. 


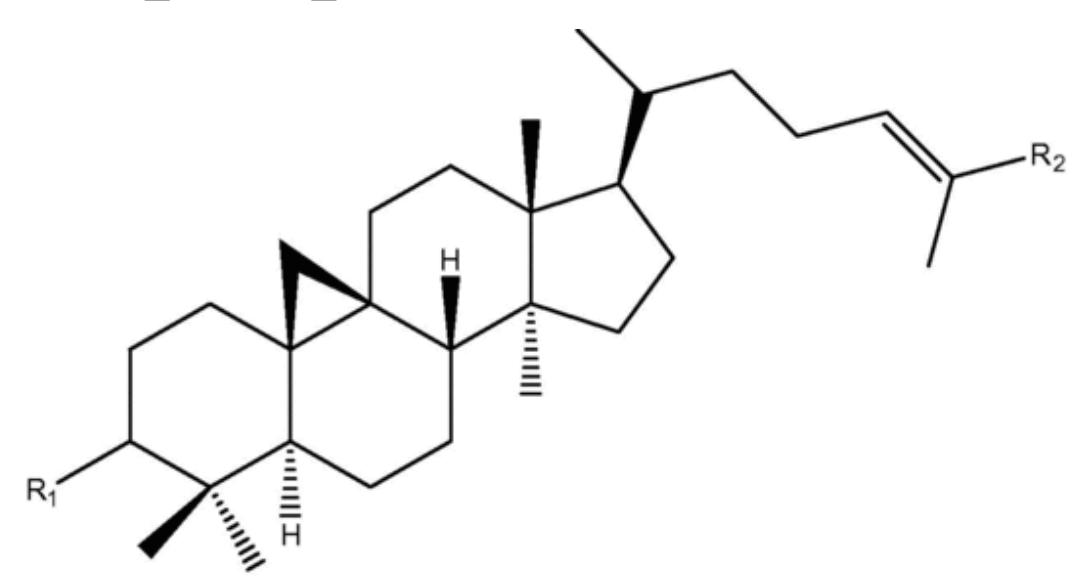

$$
\begin{aligned}
& \mathrm{R}_{1}=\beta-\mathrm{OH}, \mathrm{R}_{2}=\mathrm{CH}_{3} \\
& \mathrm{R}_{1}==\mathrm{O}, \mathrm{R}_{2}=\mathrm{COOH} \\
& \mathrm{R}_{1}=\beta-\mathrm{OH}, \mathrm{R}_{2}=\mathrm{COOH} \\
& \mathrm{R}_{1}=\alpha-\mathrm{OH}, \mathrm{R}_{2}=\mathrm{COOH}
\end{aligned}
$$

\section{Cycloartenol (17)}

Mangiferonic acid (18)

Mangiferolic acid (20)

Isomangiferolic acid (22)

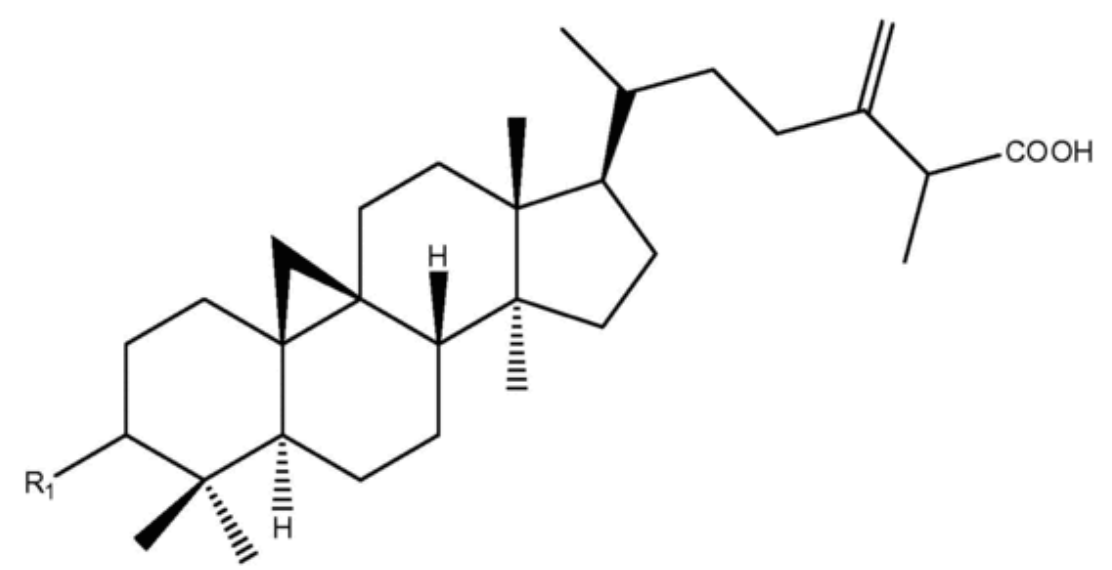

$\mathrm{R}_{1}==\mathrm{O}$, Ambonic acid (19)

$\mathrm{R}_{1}=\beta-\mathrm{OH}$, Ambolic acid (21)

Fig. 2 Structures of cycloartane triterpenes in Cameroonian propolis.

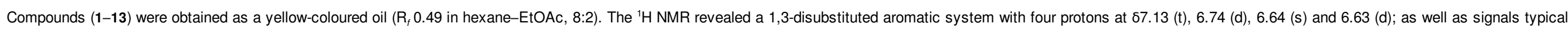

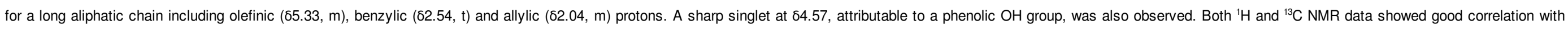




\section{elsevier_PHYTO_10940}

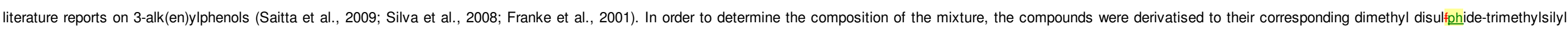

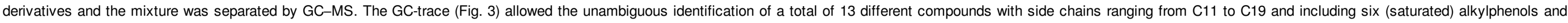

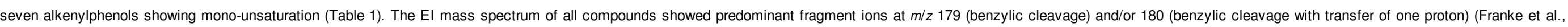

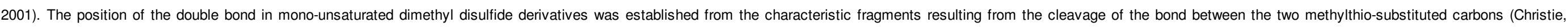

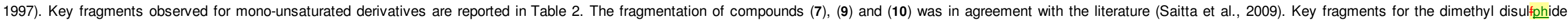

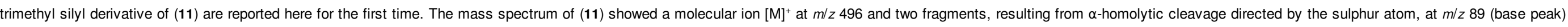

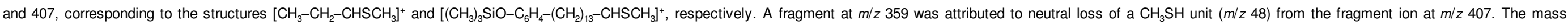

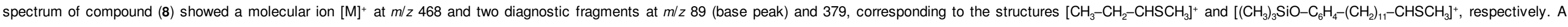

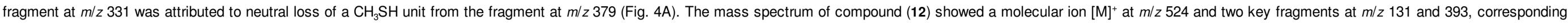

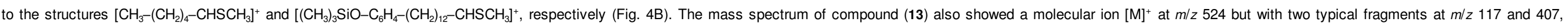

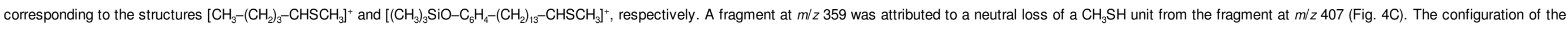

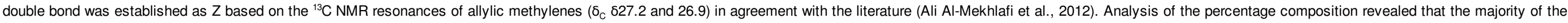

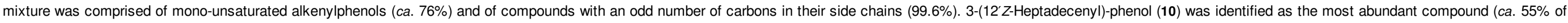
the mixture) (Table 1).

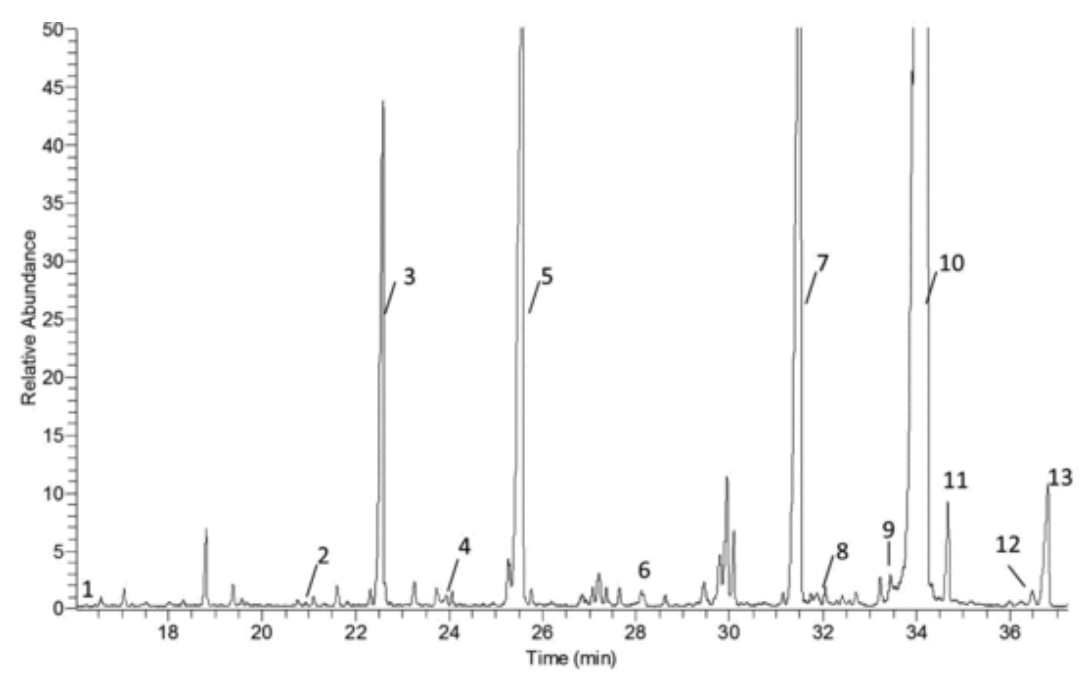

Fig. 3 GC-MS trace of alk(en)ylphenols (dimethyldisulfphide-trimethylsilyl derivatives) in Cameroonian propolis. The identity of peaks is reported in Table 1.

Table 1 Percentage composition of identified alk(en)ylphenols (1-13) in Cameroonian propolis. ${ }^{\text {a }}$

\begin{tabular}{|c|c|c|}
\hline Compound name & Range (\%) & Mean \pm SD $(\%)$ \\
\hline 3-Undecyl phenol (1) & $0.07-0.09$ & $0.09 \pm 0.01$ \\
\hline 3-Tetradecylphenol (2) & $0.07-0.10$ & $0.09 \pm 0.01$ \\
\hline 3-Pentadecylphenol (3) & $7.47-8.72$ & $8.06 \pm 0.63$ \\
\hline 3-Hexadecylphenol (4) & $0.16-0.19$ & $0.17 \pm 0.02$ \\
\hline
\end{tabular}




\section{elsevier_PHYTO_10940}

\begin{tabular}{|c|c|c|}
\hline 3-Heptadecylphenol (5) & $14.34-15.87$ & $15.16 \pm 0.77$ \\
\hline 3-Nonadecylphenol (6) & $0.26-0.32$ & $0.28 \pm 0.04$ \\
\hline 3-(10'Z-Pentadecenyl)-phenol (7) & $14.79-17.0$ & $16.16 \pm 1.20$ \\
\hline 3-(12'Z-Pentadecenyl)-phenol (8) & $0.28-0.35$ & $0.31 \pm 0.03$ \\
\hline 3-(8'Z-Heptadecenyl)-phenol (9) & $0.63-0.80$ & $0.74 \pm 0.10$ \\
\hline 3-(12'Z-Heptadecenyl)-phenol (10) & $54.48-55.58$ & $54.98 \pm 0.56$ \\
\hline 3-(14'Z-Heptadecenyl)-phenol (11) & $1.10-1.36$ & $1.25 \pm 0.13$ \\
\hline 3-(13'Z-Nonadecenyl)-phenol (12) & $0.35-0.39$ & $0.37 \pm 0.02$ \\
\hline 3-(14'Z-Nonadecenyl)-phenol (13) & $1.99-2.35$ & $2.17 \pm 0.18$ \\
\hline
\end{tabular}

${ }^{a}$ Determined on the basis of peak areas obtained by GC-MS (three replicates).

Table 2 Key fragments observed on the mass spectra of mono-unsaturated alkenylphenols (7-13) (dimethyldisulfphide-trimethylsilyl derivatives).

\begin{tabular}{|c|c|c|c|}
\hline Compound & $\mathrm{RT}(\min )$ & {$[\mathrm{M}]^{+}, \mathrm{m} / \mathrm{z}(\%)$} & Key fragment ions, $m / z(\%)$ \\
\hline (7) & 31.51 & $468(2)$ & $351(30), 303(6), 179(70), 117(85)$ \\
\hline (8) & 32.07 & $468(2)$ & $379(6), 331(5), 180(55), 179(50), 89(100)$ \\
\hline (9) & 33.43 & $496(3)$ & $323(24), 275(14), 179(60), 173(60)$ \\
\hline (10) & 34.20 & $496(3)$ & 379 (35), 331 (20), 179 (85), $117(100)$ \\
\hline (11) & 34.67 & $496(2)$ & 407 (9), 359 (9), 179 (44), 89 (100) \\
\hline (12) & 36.47 & $524(4)$ & $393(26), 179(32), 131(50)$ \\
\hline (13) & 36.80 & $524(4)$ & $407(20), 359(10), 179(50), 117(95)$ \\
\hline
\end{tabular}




\section{elsevier_PHYTO_10940}
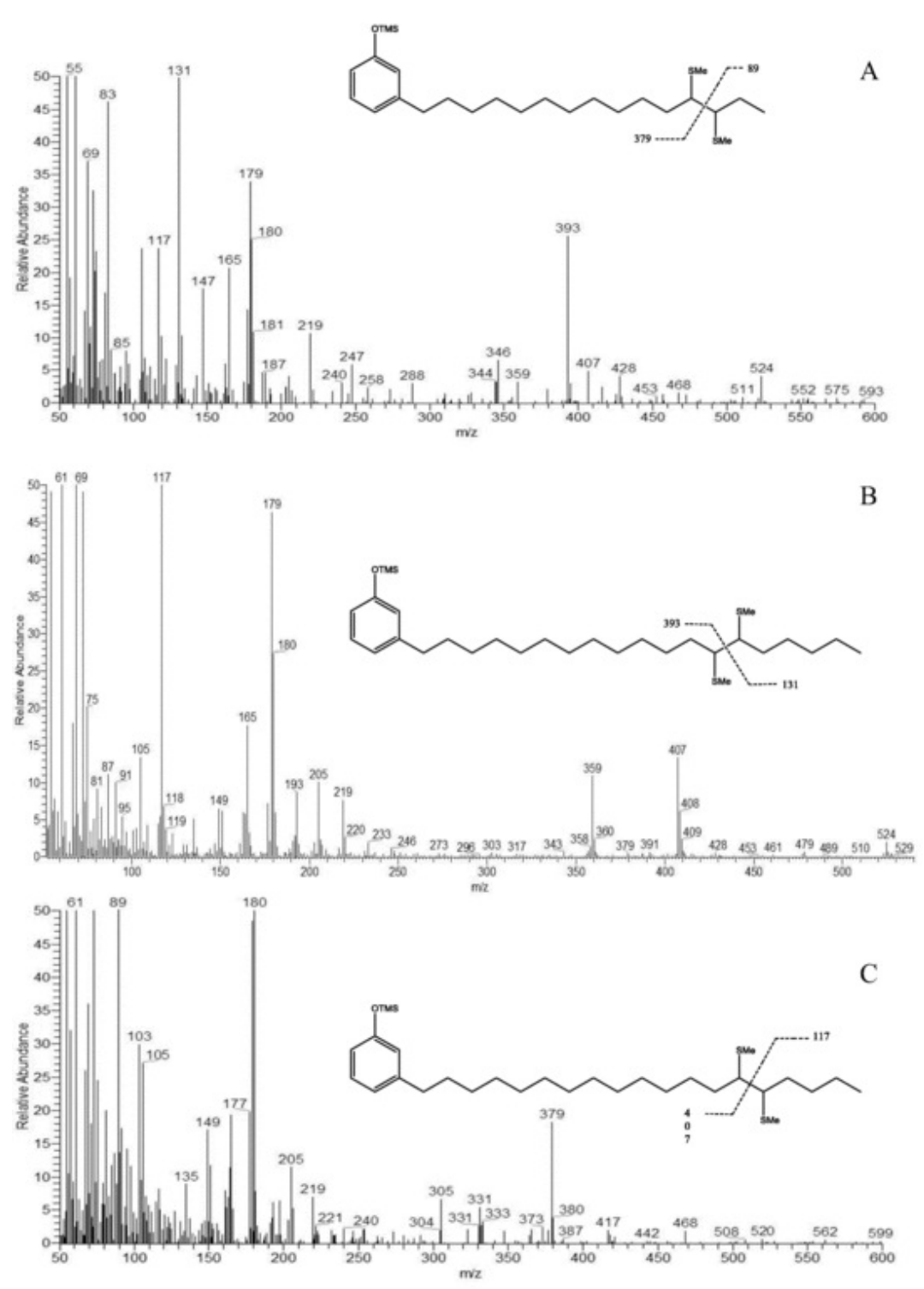

Fig. 4 El mass spectra including key fragmentation of (A) compound (8); (B) compound (12); (C) compound (13) (dimethyldisulfphide-trimethylsilyl derivatives).

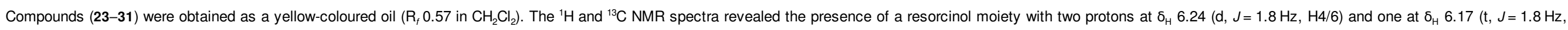

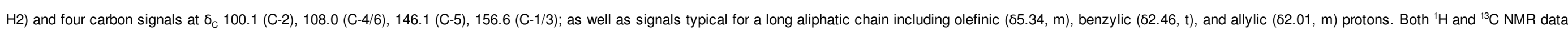

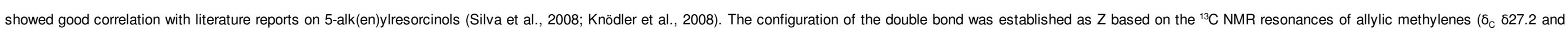

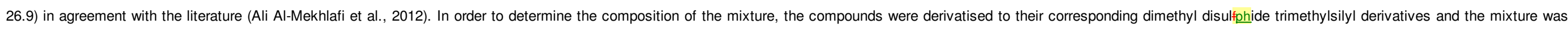

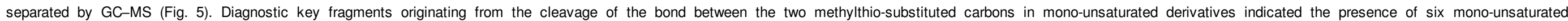




\section{elsevier_PHYTO_10940}

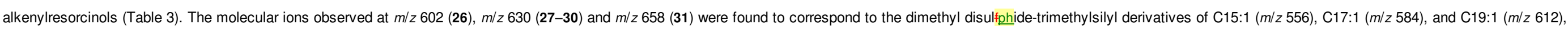

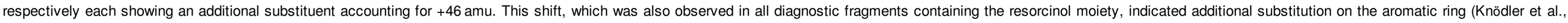

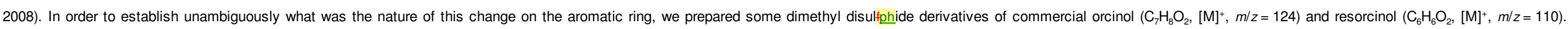

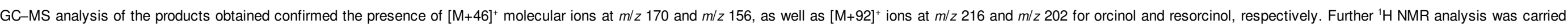

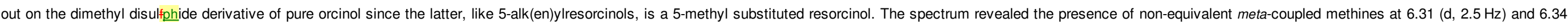

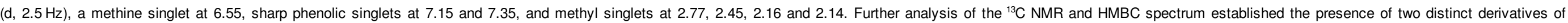

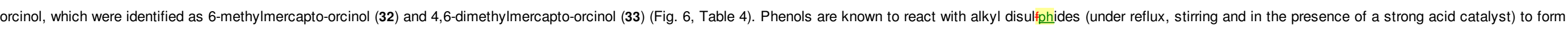

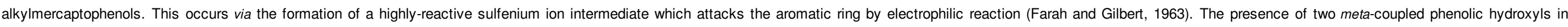

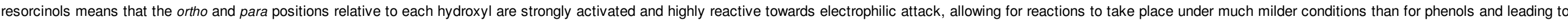

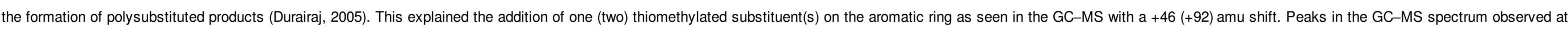
$\mathrm{m} / \mathrm{z}$ 510, 524 and 538 were subsequently identified as C15:0 (23), C16:0 (24) and C17:0 (25) methylmercaptoresorcinol derivatives.

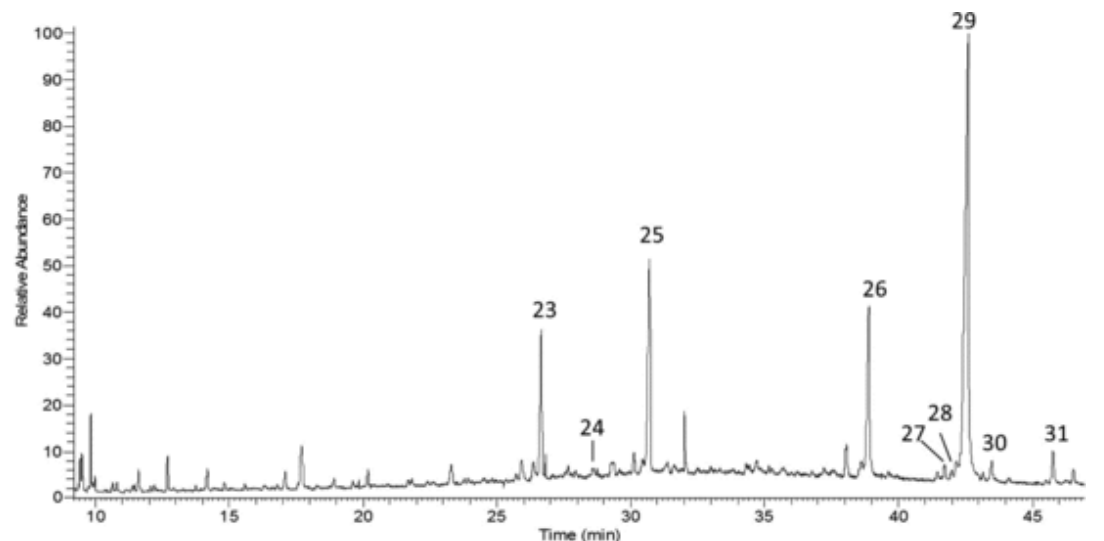

Fig. 5 GC-MS trace of alk(en)ylresorcinols (dimethyldisulffhide-trimethylsilyl derivatives) in Cameroonian propolis. The identity of peaks is reported in Table 3.

Table 3 Key fragments observed on the mass spectra of mono-unsaturated alkenylresorcinols (26-31) (dimethyldisulfphide-trimethylsilyl derivatives)

\begin{tabular}{|c|c|c|c|}
\hline Compound & $\mathrm{RT}(\min )$ & {$[M+46]^{+}, m / z(\%)$} & Key fragment ions, $m / z(\%)$ \\
\hline (26) & 38.89 & $602(100)$ & 485 (60), 268 (35), 117 (35) \\
\hline (27) & 41.72 & $630(85)$ & 457 (70), 267 (35), $173(95)$ \\
\hline (28) & 42.16 & $630(70)$ & 499 (45), 268 (55), 131 (55) \\
\hline (29) & 42.51 & $630(87)$ & 513 (100), 267 (55), 117 (85) \\
\hline (30) & 43.49 & $630(90)$ & 541 (55), 268 (55), 89 (90) \\
\hline (31) & 45.78 & $658(90)$ & $541(85), 267(82), 117(75)$ \\
\hline
\end{tabular}




\section{elsevier_PHYTO_10940}

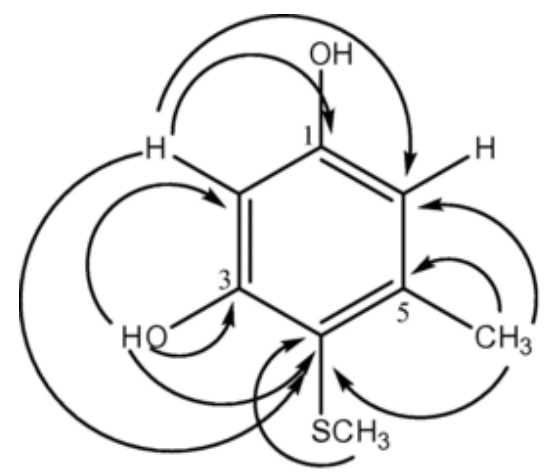

(32)

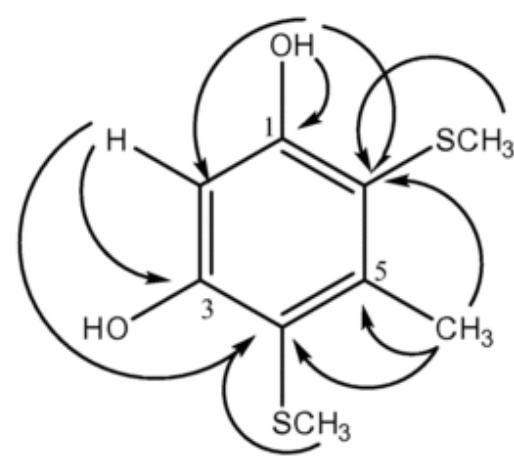

(33)

Fig. 6 Important HMBC correlations (H C) for compounds (32) and (33).

Table 4 NMR data for orcinol and compounds (32) and (33) in $\mathrm{CDCl}_{3}{ }^{\text {a }}$

\begin{tabular}{|c|c|c|c|c|c|}
\hline \multirow[t]{2}{*}{ Position } & \multirow{2}{*}{$\begin{array}{c}\text { Orcinol } \\
{ }^{1} \mathrm{H}\end{array}$} & \multicolumn{2}{|c|}{ (32) } & \multicolumn{2}{|c|}{ (33) } \\
\hline & & ${ }^{1} \mathrm{H}$ & ${ }^{13} \mathrm{C}$ & ${ }^{1} \mathrm{H}$ & ${ }^{13} \mathrm{C}$ \\
\hline 1 & - & - & 158.2 & - & 159.0 \\
\hline 2 & $6.16 \mathrm{~s}$ & $6.34 d(2.5)$ & 99.2 & $6.55 \mathrm{~s}$ & 98.4 \\
\hline 3 & - & - & 157.3 & & 159.0 \\
\hline 4 & $6.23 \mathrm{~s}$ & $6.31 \mathrm{~d}(2.5)$ & 109.5 & - & 112.3 \\
\hline 5 & - & - & 144.6 & - & 148.4 \\
\hline 6 & $6.23 \mathrm{~s}$ & - & 111.7 & - & 112.5 \\
\hline $\mathrm{Me}$ & $2.24 \mathrm{~s}$ & $2.45 \mathrm{~s}$ & 20.8 & $2.77 \mathrm{~s}$ & 19.4 \\
\hline $\mathrm{SMe}$ & & $2.15 \mathrm{~s}$ & 18.7 & $2.16 \mathrm{~s}$ & 18.7 \\
\hline 1/3-OH & & $7.15 \mathrm{~s}$ & & $7.35 \mathrm{~s}$ & \\
\hline
\end{tabular}

a ${ }^{1} \mathrm{H}(400 \mathrm{MHz})$ and ${ }^{13} \mathrm{C}(100 \mathrm{MHz})$; assignments were established by DEPT 135 and $\mathrm{HMBC}$ data.

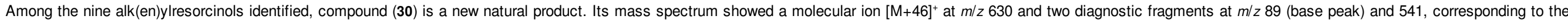

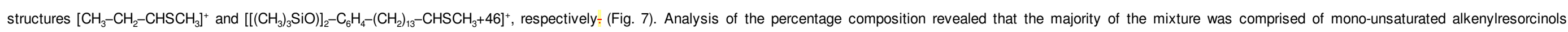
$(77.8 \%)$ and of compounds with an odd number of carbons in their side chains (99.3\%). 5-(12' Z-Heptadecenyl)-resorcinol (29) was identified as the most abundant compound (ca. 58\% of the mixture) (Table 5). 


\section{elsevier_PHYTO_10940}

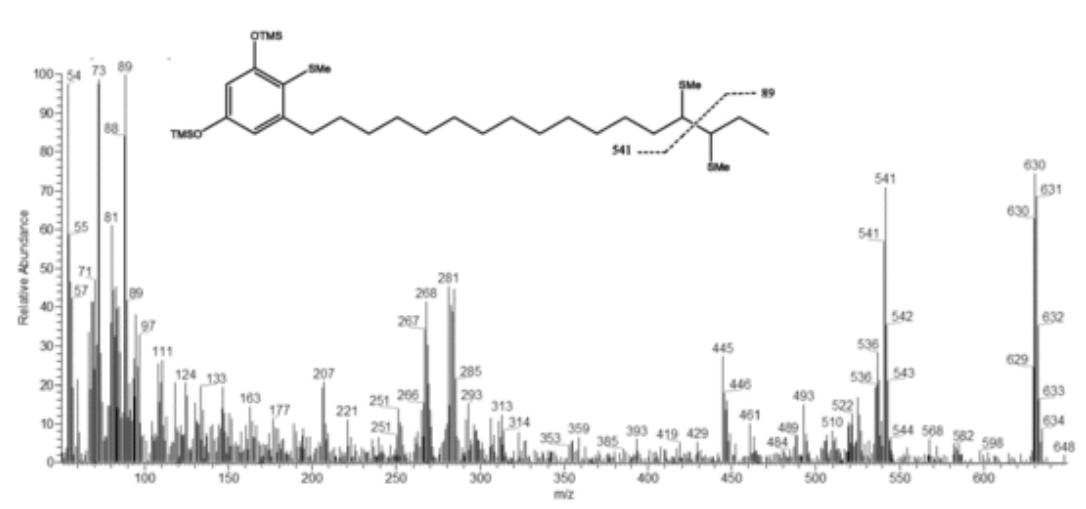

Fig. 7 El mass spectra including key fragmentation of compound (30) (dimethyldisulfohide-trimethylsilyl derivative).

Table 5 Percentage composition of identified alk(en)ylresorcinols (23-31) in Cameroonian propolis. ${ }^{a}$

\begin{tabular}{|c|c|c|}
\hline Compound name & Range (\%) & Mean \pm SD $(\%)$ \\
\hline 5-Pentadecy|resorcinol (23) & $6.89-9.03$ & $7.64 \pm 1.20$ \\
\hline 5-Hexadecylresorcinol (24) & $0.53-0.86$ & $0.67 \pm 0.17$ \\
\hline 5-Heptadecylresorcinol (25) & $13.25-14.66$ & $13.86 \pm 0.73$ \\
\hline 5-(10'Z-Pentadecenyl)-resorcinol (26) & $13.86-14.1$ & $13.99 \pm 0.12$ \\
\hline 5-(8'Z-Heptadecenyl)-resorcinol (27) & $1-1.17$ & $1.08 \pm 0.08$ \\
\hline 5-(11'Z-Heptadecenyl)-resorcinol (28) & $0.4-0.64$ & $0.52 \pm 0.12$ \\
\hline 5-(12'Z-Heptadecenyl)-resorcinol (29) & $55.89-59.1$ & $57.97 \pm 1.81$ \\
\hline 5-(14'Z-Heptadecenyl)-resorcinol (30) & $1.46-1.68$ & $1.58 \pm 0.11$ \\
\hline 5-(14'Z-Nonadecenyl)-resorcinol (31) & $2.45-2.98$ & $2.69 \pm 0.27$ \\
\hline
\end{tabular}

a Determined on the basis of peak areas obtained by GC-MS (three replicates).

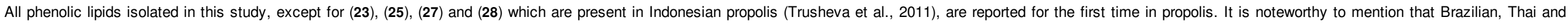

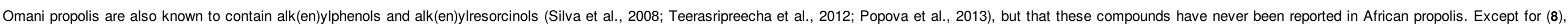

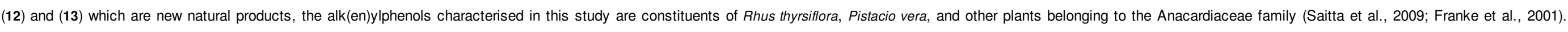

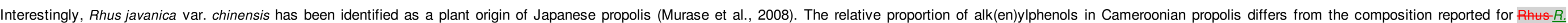

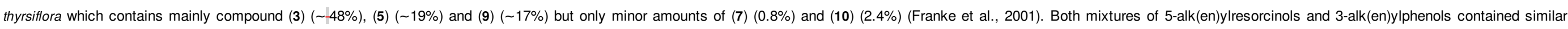

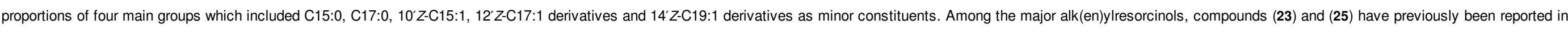

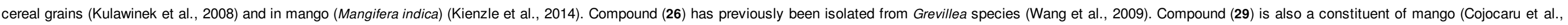
1986).

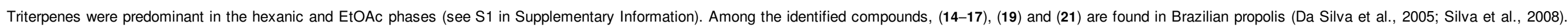

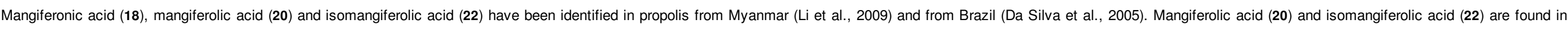

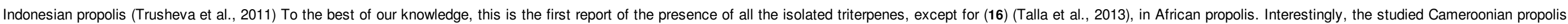




\section{elsevier_PHYTO_10940}

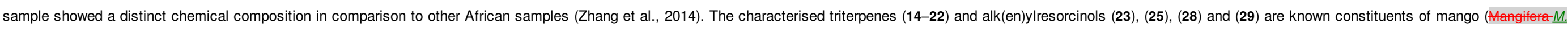

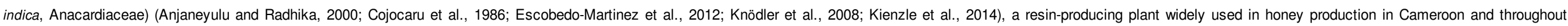

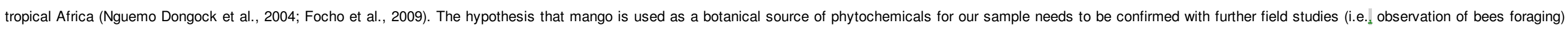
and comparative analyses of the chemical composition, if collected, of mango resin and of propolis used in local hives.

\section{Experimental}

\subsection{General experimental procedures}

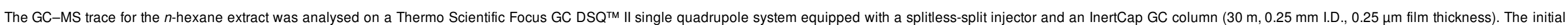

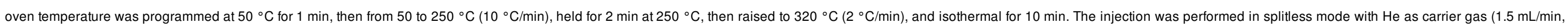
constant rate). The ion source temperature was set at $250^{\circ} \mathrm{C}$. The mass spectrometer was used in El mode $(70 \mathrm{eV})$ and operated from 50 to 800 amu with full scan detection each second.

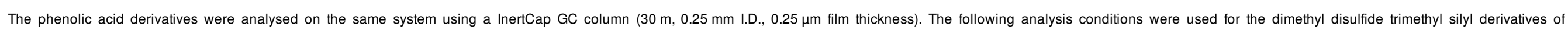

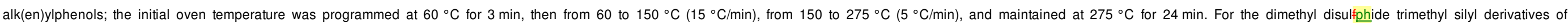

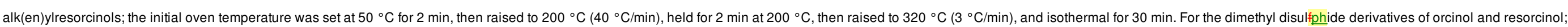

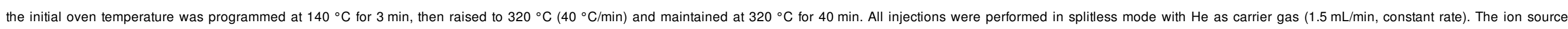

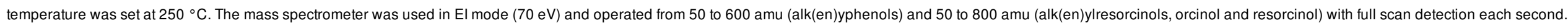

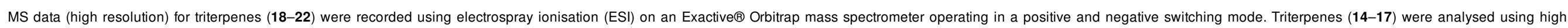

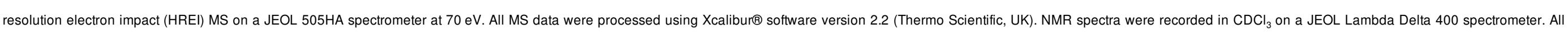
spectra were referenced on the residual solvent peaks and processed using Mestre Nova® (MNova) software version 8.0.0 (Mestrelab Research SL, Spain).

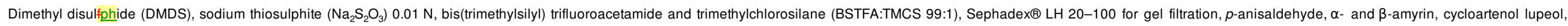

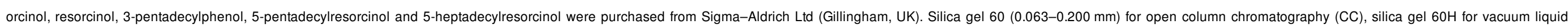

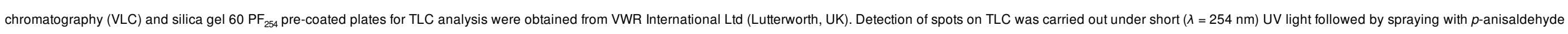
sulphuric acid reagent and heating until colours appeared.

\subsection{Propolis material}

The sample of propolis originating from Sel and Fio C.G beekeepers, Nguabum-Konene (Menchum division, North-West region, Cameroon) was supplied by the Apiceutical Research Centre.

\subsection{Extraction and isolation}

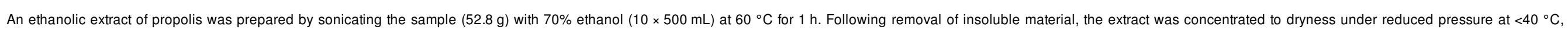

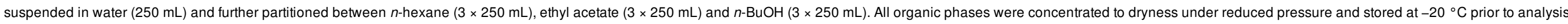

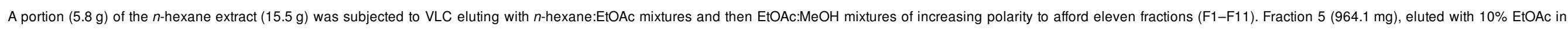

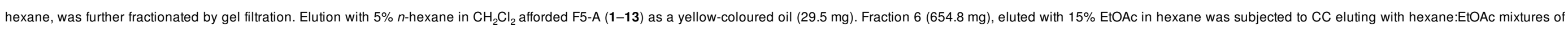

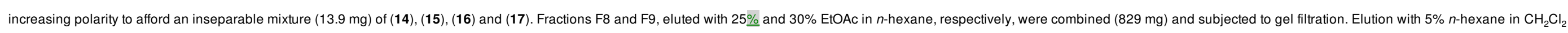

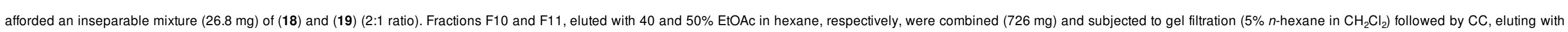
hexane:EtOAc mixtures of increasing polarity, to afford a (1:1) mixture $(6.7 \mathrm{mg})$ of $(\mathbf{2 0})$ and $(\mathbf{2 1})$.

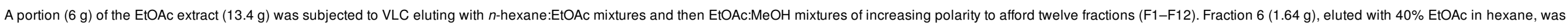
further fractionated by gel filtration eluting with $5 \% n$-hexane in $\mathrm{CH}_{2} \mathrm{Cl}_{2}$ followed by $100 \% \mathrm{CH}_{2} \mathrm{Cl}_{2}$ to yield a mixture $(7.2 \mathrm{mg})$ of (21) and (22), along with (18) (13.1 $\left.\mathrm{mg}\right)$ and (20) (15.5 $\left.\mathrm{mg}\right)$ as pure compounds. 


\section{elsevier_PHYTO_10940}

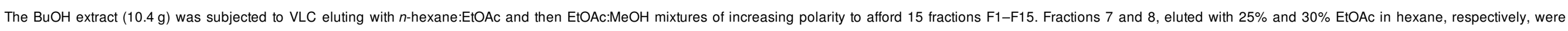
combined $(220 \mathrm{mg})$, and further fractionated by gel filtration. Elution with $\mathrm{CH}_{2} \mathrm{Cl}_{2}$ and later 10\% $\mathrm{EtOAc} \mathrm{in} \mathrm{CH}_{2} \mathrm{Cl}_{2}$ afforded $\mathrm{F} 7 / 8-\mathrm{A}(23-31)$ as a yellow-coloured oil (40 mg).

\subsubsection{Compound 14 ( $\alpha$-amyrin)}

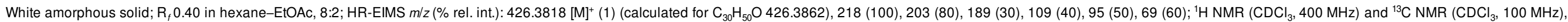
see S2 in Supplementary Information.

\subsubsection{Compound 15 ( $\beta$-amyrin)}

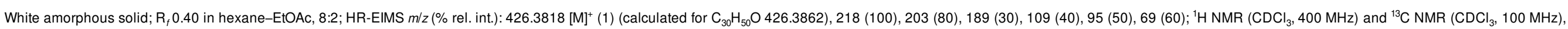
see S2 in Supplementary Information.

\subsubsection{Compound 16 (lupeol)}

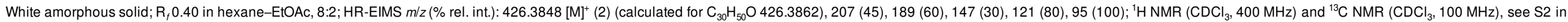
Supplementary Information.

\subsubsection{Compound 17 (cycloartenol)}

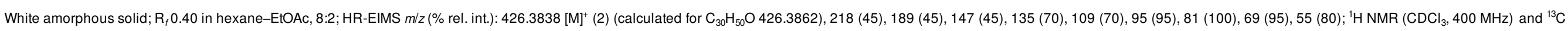
NMR $(\mathrm{CDCl} 3,100 \mathrm{MHz})$, see S3 in Supplementary Information.

\subsubsection{Compound 18 (mangiferonic acid)}

White amorphous solid; $\mathrm{R}_{f} 0.23$ in hexane-EtOAc, 8:2; HR-ESIMS: $[\mathrm{M}+\mathrm{H}]^{+} \mathrm{m} / 2455.3523$ (calculated for $\left.\mathrm{C}_{30} \mathrm{H}_{47} \mathrm{O}_{3} 455.3525\right) .{ }^{1} \mathrm{H} \mathrm{NMR}\left(\mathrm{CDCl}_{3}, 400 \mathrm{MHz}\right)$ and ${ }^{13} \mathrm{C} \mathrm{NMR}(\mathrm{CDCl} 3,100 \mathrm{MHz}$ ), see S3 in Supplementary Information.

\subsubsection{Compound 19 (ambonic acid)}

White amorphous solid; $\mathrm{R}_{f} 0.23$ in hexane-EtOAc, 8:2; HR-ESIMS: $[\mathrm{M}+\mathrm{H}]^{+} \mathrm{m} / \mathrm{z} 469.3678$ (calculated for $\left.\mathrm{C}_{31} \mathrm{H}_{49} \mathrm{O}_{3} 469.3682\right) .{ }^{1} \mathrm{H} \mathrm{NMR}\left(\mathrm{CDCl}_{3}, 400 \mathrm{MHz}\right)$ and ${ }^{13} \mathrm{C} \mathrm{NMR}(\mathrm{CDCl} 3,100 \mathrm{MHz}$ ), see S3 in Supplementary Information.

\subsubsection{Compound 20 (mangiferolic acid)}

White amorphous solid; $\mathrm{R}_{f} 0.40$ in hexane-EtOAc, $7: 3$; HR-ESIMS $[\mathrm{M}+\mathrm{H}]^{+} \mathrm{m} / \mathrm{z} 457.3674$ (calculated for $\left.\mathrm{C}_{30} \mathrm{H}_{49} \mathrm{O}_{3} 457.3682\right) ;{ }^{1} \mathrm{H} \mathrm{NMR}(\mathrm{CDCl})_{3}, 400 \mathrm{MHz}$ ) and ${ }^{13} \mathrm{C} \mathrm{NMR}(\mathrm{CDCl} 3,100 \mathrm{MHz}$ ), see $\mathrm{S} 4$ in Supplementary Information.

\subsubsection{Compound 21 (ambolic acid)}

White amorphous solid; $\mathrm{R}_{f} 0.40$ in hexane-EtOAc, $7: 3$; HR-ESIMS: $[\mathrm{M}-\mathrm{H}]^{-} \mathrm{m} / \mathrm{z} 471.3833$ (calculated for $\mathrm{C}_{31} \mathrm{H}_{51} \mathrm{O}_{3} 471.3838 .{ }^{1} \mathrm{H} \mathrm{NMR}\left(\mathrm{CDCl}_{3}, 400 \mathrm{MHz}\right.$ ) and ${ }^{13} \mathrm{C}$ NMR (CDCl3, $100 \mathrm{MHz}$ ), see $\mathrm{S} 4$ in Supplementary Information.

\subsubsection{Compound 22 (isomangiferolic acid)}

White amorphous solid; $\mathrm{R}_{f} 0.40$ in hexane-EtOAc, $7: 3$; $\mathrm{HR}$-ESIMS: $[\mathrm{M}+\mathrm{H}]^{+} \mathrm{m} / \mathrm{z} 457.3676$ (calculated for $\left.\mathrm{C}_{30} \mathrm{H}_{49} \mathrm{O}_{3} 457.3682\right) .{ }^{1} \mathrm{H} \mathrm{NMR}\left(\mathrm{CDCl}_{3}, 400 \mathrm{MHz}\right)$ and ${ }^{13} \mathrm{C} \mathrm{NMR}(\mathrm{CDCl} 3,100 \mathrm{MHz}$ ), see 44 in Supplementary Information.

\subsection{Preparation of dimethyl disulfphide and dimethyl disulfphide trimethylsilyl derivatives}

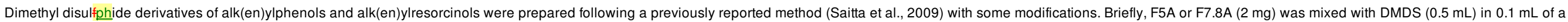

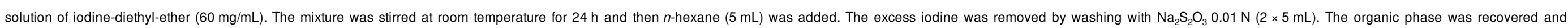

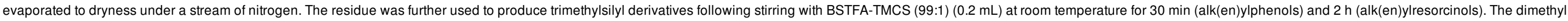




\section{elsevier_PHYTO_10940}

disulfphide trimethylsilyl derivatives obtained were dissolved in $\mathrm{CH}_{2} \mathrm{Cl}_{2}$ and analysed by GC-MS.

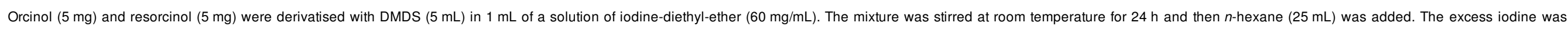

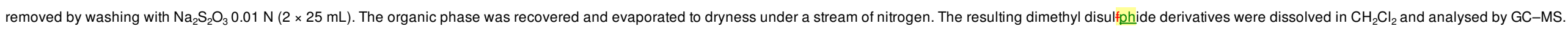

\section{Uneited referenees}

Petrat. (2010), Sn (1999) and Stasitkand Koztheck (2010).

\section{Acknowledgement}

This work was supported by the Leverhulme Trust, UK (Research Project Grant RPG-150).

\section{Appendix A. Supplementary data}

Supplementary data associated with this article can be found, in the online version, at http://dx.doi.org/10.1016/j.phytochem.2014.07.016.

\section{References}

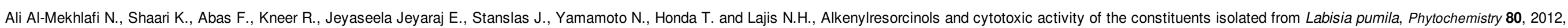
$42-49$.

Anjaneyulu V. and Radhika P., The triterpenoids and steroids form Mangifera indica Linn, Indian J. Chem. B. 39, 2000, 883-893.

Bankova V., Recent trends and important developments in propolis research, eCAM 2, 2005, 29-32.

Bankova V., Popova M., Bogdanov S. and Sabatini A.G., Chemical composition of European propolis: expected and unexpected results, Z. Naturforsch. C 57, 2002, 530-533.

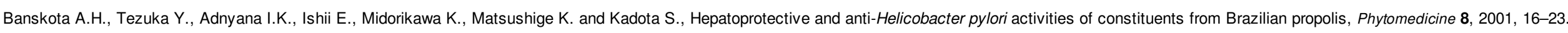

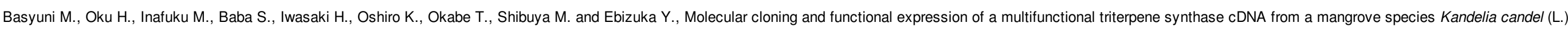

Druce, Phytochemistry 67, 2006, 2517-2524.

Borrelli F., Maffia P., Pinto L., lanaro A., Russo A., Capasso F. and Ialenti A., Phytochemical compounds involved in the anti-inflammatory effect of propolis extract, Fitoterapia 73 (Suppl. 1), 2002 , S53-S63.

Castaldo S. and Capasso F., Propolis, an old remedy used in modern medicine, Fitoterapia 73 (Suppl. 1), 2002, S1-S6.

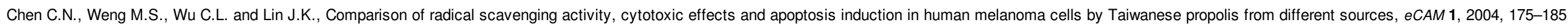

Christie W.W., Dimethyl disulphide derivatives in fatty acid analysis, Lipid Technol. 9, 1997, 17-19.

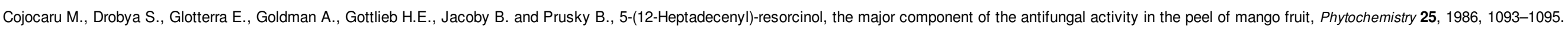

Cuesta Rubio O., Frontana-Uriba B.A., Ramirez-Apan T. and Cardenas J., Polyisoprenylated benzophenones in Cuban propolis; biological activity of nemorosone, Z. Naturforsch. C 57, 2002, 372-378.

Da Silva M.S.S., Cito A.M.G.L., Chaves M.H. and Lopes J.A.D., Cycloartane triterpenoids of propolis from Teresina-PI, Quim. Nova 28, $2005,801-804$.

Durairaj R.B., Resorcinol derivatives, In: Resorcinol: Chemistry, Technology and Applications, 2005, Springer-Verlag; Berlin.

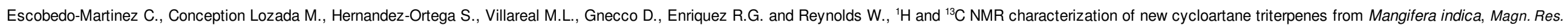
Chem. 50, 2012, 52-57.

Farah B.S. and Gilbert E.E., Alkylmercaptophenols by sulfenylation of phenols, J. Org. Chem. 28, 1963, 2807-2809. 


\section{elsevier_PHYTO_10940}

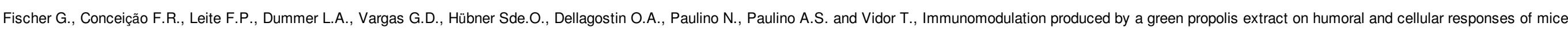
immunized with SuHV-1, Vaccine 25, 2007, 1250-1256.

Focho D.A., Newu M.C., Anjah M.G., Nwana F.A. and Ambo F.B., Ethnobotanical survey of trees in Fundong, Northwest region, Cameroon, J. Ethnobiol. Ethnomed. 5, 2009, 17-21.

Franke K., Masaoud M. and Schmidt J., Cardanols from leaves of Rhus thyrsiflora, Planta Med. 67, 2001, 477-479.

Hegazi A.G., Abd El Hady F.K. and Abd Allah F.A., Chemical composition and antimicrobial activity of European propolis, Z. Naturforsch. C 55, 2000, 70-75.

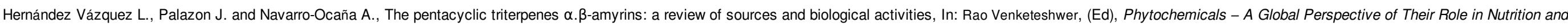
Health, 2012, In Tech Publisher; Rijeka, Croatia.

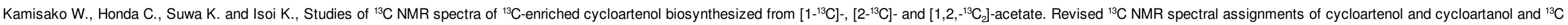
NMR spectral support for the generally accepted skeleton formation mechanism of cycloartenol, Magn. Res. Chem. 25, 1987, 683-687.

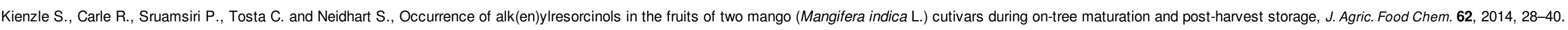

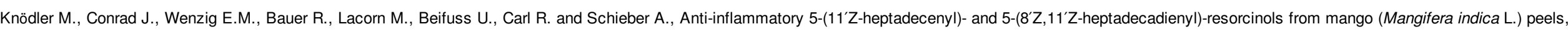

Phytochemistry 69, 2008, 988-993.

Kulawinek M., Jaromin A., Kozubek A. and Zarnowski R., Alkylresorcinols in selected polish rye and wheat cereals and whole-grain cereal products, J. Agric. Food Chem. 56, $2008,7236-7242$.

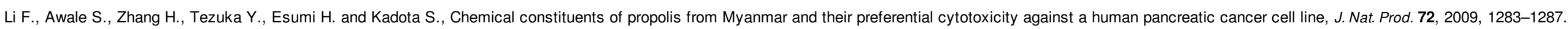

Mahato S.B. and Kundu A.P., ${ }^{13} \mathrm{C}$ NMR spectra of pentacyclic triterpenoids - a compilation and some salient features, Phytochemistry 37, 1994, 1517-1575.

Marcucci M.C. and Bankova V.S., Chemical composition, plant origin and biological activity of Brazilian propolis, Curr. Top. Phytochem. 2, 1999, 115-123.

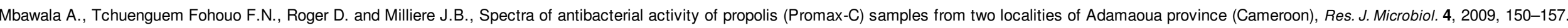

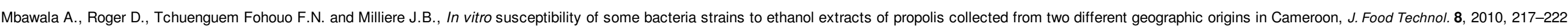

Murase M., Kato M., Sun A., Ono T., Nakamura J., Sato T. and Kumazawa S., Rhus javanica var. chinensis as a new plant origin of propolis from Okayama, Japan, Biosci. Biotechnol. Biochem. 72, 2008, 2782-2784.

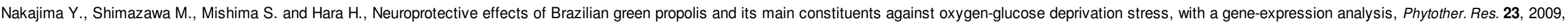

$1431-1438$.

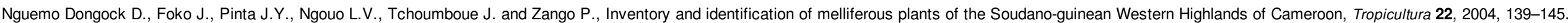

Njintang Yanou N., Tatsadjieu Ngoune L., Ngakou A., Danra D. and Tchuenguem-Fohouo F.N., Antiradical activity and polyphenol content of ethanolic extracts of propolis, Int. J. Biosci. 2, 2012, 56-63.

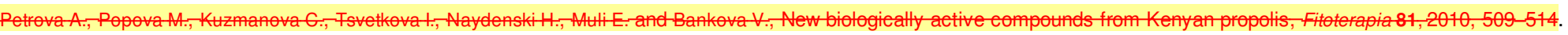

Popova M., Dimitrova R., Al-Lawati H.T., Tsvetkova I., Najdenski H. and Bankova V., Omani propolis: chemical profiling, antibacterial activity and new propolis plant sources, Chem. Cent. J. 7, 2013, 158-165.

Raghukumar R., Vali L., Watson D., Fearnley J. and Seidel V., Antimethicillin-resistant Staphylococcus aureus (MRSA) activity of 'pacific propolis' and isolated prenylflavanones, Phytother. Res. 24, $2010,1181-1187$.

Saitta M., Giuffrida D., La Torre G.L., Potorti A.G. and Dugo G., Characterisation of alkylphenols in pistachio (Pistacia vera L.) kernels, Food Chem. 117, 2009, 451-455,

Sawicka D., Car H., Borawska M.H. and Nikliński J., The anticancer activity of propolis, Folia Histochem. Cytobiol. 50, 2012, $25-37$.

Seidel V., Peyfoon E., Watson D.G. and Fearnley J., Comparative study of the antibacterial activity of propolis form different geographical and climatic zones, Phytother. Res. 22, 2008, 1256-1263. 


\section{elsevier_PHYTO_10940}

Silva M.S.S., de Lima S.G., Oliveira E.H., Lopes J.A.D., Chaves M.H., Reis F.A.M. and Cito A.M.G.L., Anacardic acid derivatives from Brazilian propolis and their antibacterial activity, Eclet. Quim. 33, 2008, 53-58.

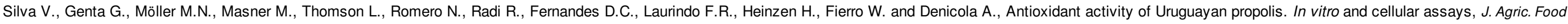
Chem. 59, 2011, 6430-6437.

Snewin V.A., Gares M.P., Gaora P.O., Hasan Z., Brown I.N. and Young D.B., Assessment of immunity to myeobacterial infection with lueiferase reporter construets, Infect. Immun. 67, 1999, 4586-4593.

Stasiuk M.-and Kozubeck A., Biologieal aetivity of phenolie lipids, Gell. Mol. Life Sei.67,2010, 841-860.

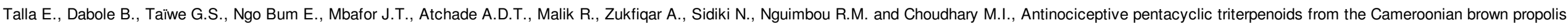

Pharmacologia 4, 2013, 218-227.

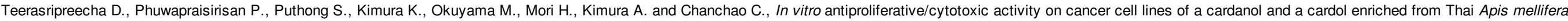
propolis, BMC Complement. Altern. Med. 12, 2012, 27-43.

Thanakijcharoenpath W. and Theanphong O., Triterpenoids from the stem of Diospyros glandulosa, Thai J. Pharm. Sci. 31, 2007, 1-8.

Trusheva B., Popova M., Naydenski H., Tsvetkova I., Rodriguez J.G. and Bankova V., New polyisoprenylated benzophenones from Venezuelan propolis, Fitoterapia 75, $2004,683-689$.

Trusheva B., Popova M., Koendhori E.B., Tsvetkova I., Naydenski C. and Bankova V., Indonesian propolis: chemical composition, biological activity and botanical origin, Nat. Prod. Res. 25, 2011 , 606-613.

Wang H., Leach D.N., Forster P.I., Thomas M.C., Blanksby S.J. and Waterman P.G., Prenylated bisresorcinols from Grevillea floribunda, Phytochem. Lett. 2, $2009,41-45$.

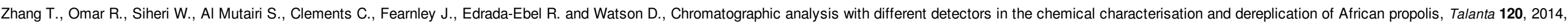
181-190.

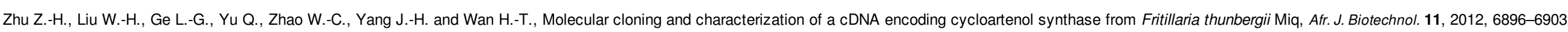

\section{Appendix A. Supplementary data}

Multimedia Component 1

Supplementary data 1

\section{Graphical abstract}

Chemical investigation of Cameroonian propolis led to the isolation of twenty-two phenolic lipids (including four new structures) along with nine triterpenes.
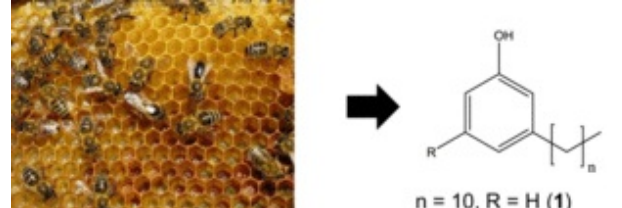

$n=10, R=H(1)$

$n=14, R=H(3) ; R=O H(23) \quad n_{1}=11, n_{2}=1, R=H(8): R=O H(27)$

$n=15, R=H(4) ; R=O H(24) \quad n_{1}=11, n_{2}=3, R=H(10) ; R=O H(29)$

$\begin{array}{ll}n=16, R=H(5) ; R=O H(25) & n_{1}=13, n_{2}=1, R=H(11) ; R=O H(30) \\ n=18, R=H(6) & n_{1}=12, n_{2}=4, R=H(12)\end{array}$

$n_{1}=10, n_{2}=4, R=O H(28)$

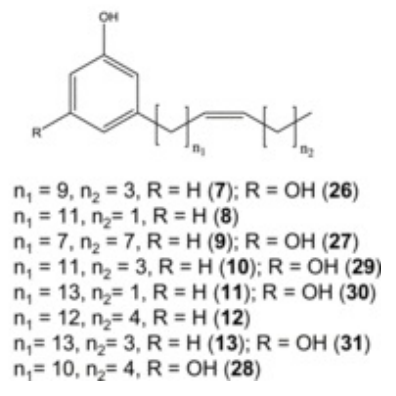




\section{elsevier_PHYTO_10940}

Highlights

- Twenty-two phenolic lipids and nine triterpenes were found in Cameroonian propolis.

- Four new phenolic lipids were characterised by GC-MS analysis.

- The most abundant phenolic lipids were identified as 3-(12'z-heptadecenyl)-phenol and 5-(12'z-heptadecenyl)-resorcinol.

- Eighteen compounds are reported for the first time in propolis.

\section{Queries and Answers}

Query: Please confirm that given name(s) and surname(s) have been identified correctly.

Answer: Confirmed.

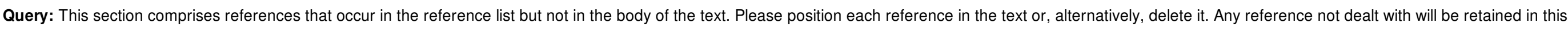
section.

Answer: These three references have now been deleted from the reference section

Query: One or more sponsor names may have been edited to a standard format that enables better searching and identification of your article. Please check and correct if necessary.

Answer: Format accepted

Query: The country names of the Grant Sponsors are provided below. Please check and correct if necessary. 'Leverhulme Trust' - 'United Kingdom'.

Answer: Country added as requested 